Health services

\section{Referral and access to children's health services}

N Simpson, P Stallard

\section{A single point of entry model}

T he Children's National Service Framework has identified access as a core theme and aims to develop standards to ensure better access and smoother progression in the provision of services. ${ }^{1}$ The need for better coordination was highlighted by the Bristol Children's Enquiry which recommended that "there must be a greater integration of primary, community, acute and specialist health care for children ... so that services for children in the future are better integrated and organised around the needs of children and their families" (recommendation 177). ${ }^{2}$ This theme also underpins the development of managed clinical networks which aim to ensure that high quality effective services are equitably provided and coordinated around the needs of the child rather than being constrained by organisational or professional boundaries. $^{34}$

At present children's health services may be hosted and managed by different organisations. Comprehensive commissioning and service development can be problematic, as it is often difficult to create an overview of local health needs and service utilisation. Information is held in different forms, in different databases, and by different services.

The provision of children's services is also complex, being delivered by a number of different professionals, at a range of levels and in different locations. Access to these services is often by a number of independent referral routes which may differ in terms of entry and urgency criteria, who is eligible to refer, and work to different geographical boundaries. Poor communication and coordination between services can lead to duplication of work or confusion about who is responsible for meeting which particular part of a child's or family's needs.

From a referrer's perspective a range of referral pathways can be a source of confusion; particularly where there are multiple needs or where the most appropriate service to address a child's needs is not clear. Navigating such a complex system can be both frustrating and time consuming for referrers, children, and their families.

\section{A SINGLE POINT OF ENTRY}

A possible response to the need to simplify and streamline access to children's health services, particularly the transition from primary to secondary care, is to establish a comprehensive single point of access for all referrals. There are few published papers on the management of referrals from primary to secondary care services, ${ }^{5-7}$ and none that include other referral agencies; in this case, education and social care to secondary care health services.

In the autumn of 2000 a single point of entry (SPE) referral system was established in Bath and North East Somerset (BANES) which has a child population (0-15 years) of approximately 30000 . Although the area is now served by one PCT co-terminus with local authority education and social services departments, secondary child health services are provided by four trusts.

The need to address equity of access and co-ordination issues through a single point of entry with improved management of transition was highlighted in the conclusion of a review of children's health services undertaken by Avon Health Authority in 1998. The implementation of the SPE was supported by significant political commitment from the old health authority and with financial resources to employ a part-time project manager for one year to facilitate the process. A multi-agency implementation group, health professional project group, and an information technology working group were established. Meetings with a wide range of professionals and a locality away day were organised to sell the vision and shape the specification. Discussion with service providers about eligibility criteria and development of documentation and processes were undertaken.

Figure 1 shows a schematic representation of the SPE. There is a single generic referral form (paper and electronic versions available), which contains patient and referrer information plus a menu of available services (see fig 1).

Paediatrics includes both "hospital and community" services. Referrals are sent to the SPE administrator who logs each on a central database and then applies a set of decision rules (developed by a multi-professional team) to determine how the referral is processed. Those that specify a single service (or specified combination, for example, speech and language therapy and hearing assessment) are "fast tracked" (within one working day) to the service specified by post or fax. An acknowledgement letter is generated to the referrer, parent, and GP if not the referrer, which includes the contact address and telephone number of the service(s) concerned. The SPE administrator is a non-clinical role. Clinical advice/support for any specific queries that might arise is available on a daily basis through the "on-call" paediatrician in the community child health department. GPs are able to access the same day assessment clinic for urgent acute referrals via the on-call hospital registrar.

If the referrer is uncertain which service is required, multiple services are requested, or there have been previous referrals in the proceeding year (excluding hearing assessments), the referral is brought forward to the intake team. This is a multi-professional group (including paediatrician, clinical psychologist, speech and language therapist, occupational therapist or physiotherapist, school nurse, learning/ behaviour support nurse, health visitor, and SPE administrator) that meets twice a month for up to an hour. Referrals are discussed and decisions made about the most appropriate service(s) to forward the referrals to.

\section{PRELIMINARY EVALUATION}

Formal evaluation of changes in referral patterns and flows was not feasible because comprehensive data were not available before implementation of the SPE model. However, an analysis of total referrals over the first two years of the SPE (fig 2) highlight that the number captured by the system has increased from 2003 in 2001 to 2293 in 2002.

The evolution and development of the SPE over the past two years has been influenced by feedback from referrers and service providers. A survey of referrers, secondary care providers, and the intake team in the first year showed much support for the model.

Perceived benefits included: simpler system, more efficient, and improved coordination and communication. Relatively few difficulties were identified but 


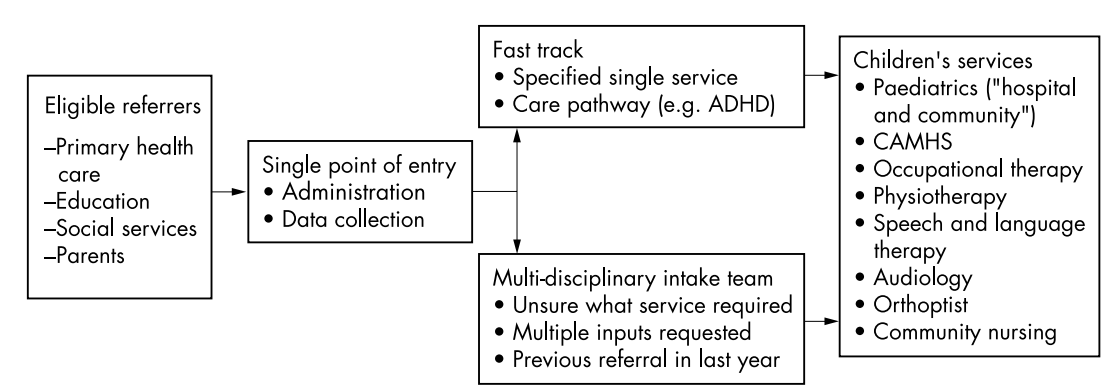

Figure 1 Schematic diagram of single point of entry model.

included: wanting access to more secondary services (for example, paediatric ENT, dermatology, and orthopaedics), possible delays, training for referrers, and the need for an electronic version of the SPE form. These issues have, or are, being addressed as part of the ongoing development of the system, some of which are discussed below.

\section{BENEFITS, ISSUES, AND CONTROVERSIES}

Facilitating a multi-professional/ agency approach to the development and provision of children's services

The process of implementing and developing the SPE has facilitated a greater understanding of professional roles and the challenges that each is facing (for example, waiting lists). It has also encouraged a shared ownership of difficult issues and ways in which they can be addressed, for example, the development of an integrated care pathway for children with attention deficit hyperactivity disorder.

Improved management of referrals Discussions of referrals where there have been previous referrals in the preceding year has meant that where appropriate the new referral is passed to the service currently involved, rather than accessing another service. Where multiple options have been requested the intake team has rationalised a significant portion, for example, suggesting school nurse assessment and support with referral if needed. Alternatively additional referrals may be generated to expedite the assessment process and save the "paper chase" whereby a family may, for example, wait to see a paediatrician and then be referred to and wait for a speech and language therapy assessment.

\section{Who are eligible referrers?}

The introduction of the SPE highlighted significant differences between services in terms of who was an "eligible referrer". Some services would accept self/family referrals, others only professionally initiated referral with some being solely limited to consultant based referrals. The underlying philosophy of the SPE was to provide not only a simpler route to children's health services but to allow greater referrer access. Referrals from primary care were therefore accepted from GPs, health visitors, school nurses, social workers, teachers, and in the case of CAMHS and Speech and Language therapy, self/family referrals. Opening the SPE to a wider range of potential referrers was initially controversial, raising the issue of whether appropriate help or assessment had been provided in primary care and concern about whether referrals from some groups, for example, direct from

\section{The postal service-a metaphor for the SPE model}

- Anyone can write a letter and post it in a letter box.

- Letter boxes in a defined area feed into one sorting office.

- There is an emphasis on efficient onward delivery to the correct address.

- If a letter is not clearly addressed, it can either be returned to the sender, or opened and checked to see if it is clear where is should be forwarded to.

- If the letter is inadvertently sent to the wrong address, the letter can be put back in a letter box for resorting.

- If a letter is urgent there are options for express delivery with systems to trace where letters are in the system.

- Usually one receives an acknowledgement or reply to one's letter.

health visitors, would be appropriate. The response to this has been to provide feedback, guidance, and training.

Health or multi-agency SPE for children's services?

The SPE was originally developed to provide a single route from primary care to secondary child health services. There were discussions about social services and education representation at the intake team, but it was agreed to keep the focus on secondary health services initially and see how the system went. Since then schools have approached the local education authority (LEA) to see whether a similar model could be set up and used to access secondary level support services from the LEA.

\section{"Health service need" or an opportunity to bypass shortfalls in educational provision?}

A particular difficulty with some education referrals was observed, where referrals to the SPE clearly highlighted concerns that were the responsibility of Education or Social Services, to address. However, these issues are not new and in view of the difficulty collecting this information prior to the SPE it was not possible to determine whether the introduction of the SPE resulted in an increase in inappropriate referrals. Centralisation of referrals through the SPE did however make them more visible and provided a useful way of identifying and educating those who regularly made inappropriate referrals.

\section{Will the SPE delay referrals?}

The SPE was introduced to improve efficiency of referrals from primary care to secondary child health services. Referrers and providers were however initially concerned that the SPE may be a bureaucratic administration system that would simply delay the passage of referrals to the appropriate services. In response to this concern the two tiered process, summarised in fig 1 , was introduced in order to fast track referrals. Interestingly a survey of eligible referrers conducted during the first year the SPE was introduced failed to identify this as a significant concern.

Professionals bypassing the system Another concern was the degree to which the SPE restricted referrer's ability to directly refer to services or professionals of their choice. Personal knowledge about a professionals style of work, concern about "urgent referrals" being delayed, and the desire to maintain and develop closer and more direct relationships between primary care and secondary care services were important factors. This was a particular 
issue for CAMHS where a number of referrals continue to be made direct to the service and as such bypass the SPE. Our approach has not been to directly challenge this practice but instead to develop a simple administrative procedure whereby the SPE is notified of all direct referrals to CAMHS.

\section{All paediatric referrals?}

The BANES SPE model includes referrals to "hospital" as well as "community" paediatricians. Having referrals from primary care coming via the SPE allows the potential to rationalise referrals in a systematic way according to geographical area, care pathways as they develop, or by special interest. This may be even more useful in tertiary centres where referrals to tertiary specialists from primary care could be rationalised according to agreed guidelines; for example, all referrals about headaches are first seen by a general paediatrician.

\section{Generalisability}

The SPE is not a "one size fits all" model but can be implemented in a flexible way based on local needs and circumstances. The SPE model could be developed for different sized populations, include different ranges of professional groups and clinical services, and be adapted to accommodate locally developed processes and decision rules.

\section{CURRENT AND FUTURE DEVELOPMENTS}

The SPE is a dynamic evolving process that needs to adapt and be responsive to new demands. Currently there are requests from referrers to access more services via the SPE system such as paediatric specialist services (for example, paediatrics, ENT, urology, orthopaedics, and dermatology). The SPE referral form is being integrated into the web based direct GP/hospital referral system. This will fit with the SPE system allowing more efficient "delivery" of referrals to the SPE administrator and ultimately to allow forwarding by email.

The SPE has also initiated interest in developing other care pathways for children with multiple and complex needs where a number of professionals may become involved in the provision of care. This agenda of care pathway development to fit with the SPE model is limited by local and national capacity to develop and quality assure.

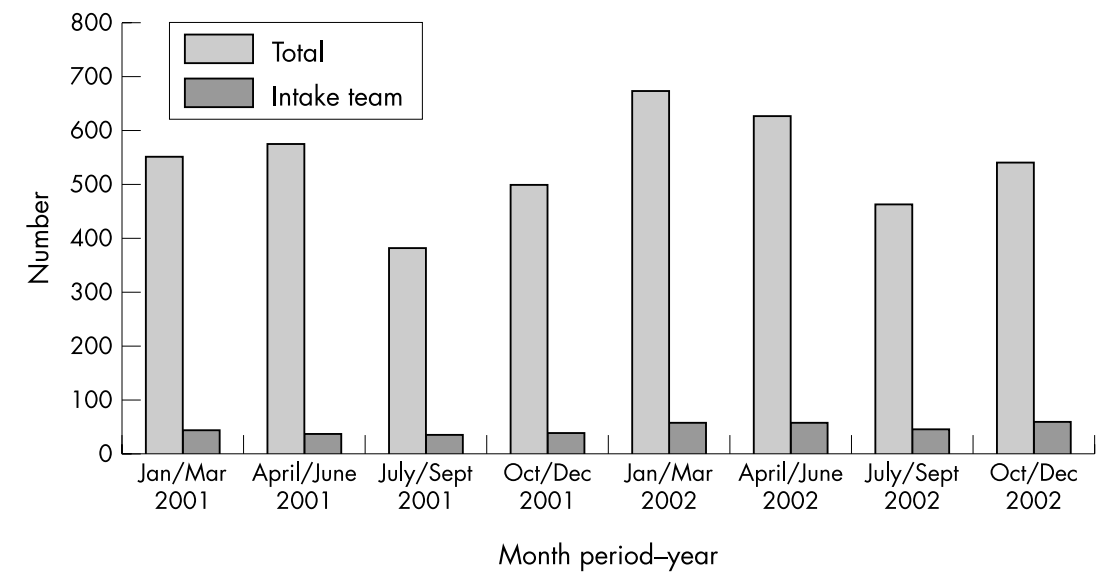

Figure 2 Total referrals (and intake team discussions) each quarter for 2001-02.

The administrative support required to operate an SPE has emerged as an important issue. Currently this is provided by 0.5 whole time equivalent administrative post that covers the processing of 100-280 referrals per month, and supporting the intake team (average of 15 referrals per month, range 6-37).

The existing computer system is limited in the information that is collected. It is not yet linked to other services IT systems, and therefore information about the child's diagnosis or whether the case is still active or closed is unknown. However, despite these limitations the data currently available provides a more comprehensive overview of referrals to children's services than was previously possible (see fig 2). The information can be used to look at referral patterns from different sources, GP practices/schools, and to different secondary services, and to identify training issues. It begins to provide better information for commissioning and allows audit of specific groups of referrals when issues are raised, for example, quality of referrals from schools.

\section{CONCLUSION}

We have described a single point of access to children's locality services in BANES, which addresses some of the current issues in providing and coordinating complex services to meet the health needs of children. The SPE has the potential to be extended and developed, and fits with current national agendas (Children's NSF, managed clinical networks, and care pathways). Regular review of the SPE process is important to enable continuous quality improvement of this system. We hope this will stimulate interest and debate at a time when health professionals need to be thinking more systemically about developing effective and efficient management of referrals, both for the quality of care for children and families, but also for themselves.

Arch Dis Child 2004;89:109-111.

doi: 10.1136/adc.2003.019794

\section{Authors' affiliations}

N Simpson, Bath NHS House, Community Child Health Department, Newbridge Hill, Bath, UK

P Stallard, Child and Family Therapy Department, Royal United Hospital, Bath BA1 3NG, UK

Correspondence to: $\operatorname{Dr} \mathrm{N}$ Simpson, Bath NHS House, Community Child Health Department, Newbridge Hill, Bath BA1 3QE, UK; neil.simpson@banes-pct.nhs.uk

\section{REFERENCES}

1 Department of Health. http://www.doh.gov.uk/ nsf/children.htm.

2 Learning from Bristol. The Bristol Royal Infirmary Inquiry. July 2001. CM 5207(I).

3 Cropper S, Hopper A, Spencer S. Managed clinical networks. Arch Dis Child 2002;87:1-4.

4 Baker C, Lorimer A. Cardiology: the development of a managed clinical network. BMJ 2000;321:1152-3.

5 Hull SA, Jones C, Tissier JM, et al. Relationship style between GPs and community mental health teams affects referral rates. $\mathrm{Br} J$ Gen Pract 2002;52(475):101-7.

6 Hull S, Harvey C, Sturdy P, et al. Do practicebased preventive child health services affect the use of hospitals? A cross-sectional study of hospital use by children in east London. $\mathrm{Br} J \mathrm{Gen}$ Pract 2000;50:31-6.

7 Gask L, Sibbald B, Creen F. Evaluating models of working at the interface between mental health services and primary care. $\mathrm{Br} J$ Psychiatry 1997;170:6-11 